\title{
Antibiofilm activity of propolis extract on Fusarium species from onychomycosis
}

\author{
Juliana Galletti ${ }^{1}$, Flávia K Tobaldini-Valerio ${ }^{1,2}$, Sónia Silva², Érika Seki Kioshima1, Larissa \\ Trierveiler-Pereira1 ${ }^{1}$, Marcos Bruschi ${ }^{3}$, Melyssa Negri ${ }^{*}{ }^{1}$ \& Terezinha Inez Estivalet Svidzinski ${ }^{1}$ \\ ${ }^{1}$ Laboratory of Medical Mycology, Departamento de Análises Clínicas e Biomedicina, Universidade Estadual de Maringá (UEM), \\ Avenida Colombo, 5790, Maringá, PR, CEP 87020-900, Brazil \\ ${ }^{2}$ CEB Centre of Biological Engineering, Universidade do Minho, Braga, Portugal \\ ${ }^{3}$ Laboratory of Research \& Development of Drug Delivery Systems, Department of Pharmacy, State University of Maringá, CEP \\ 87020-900 Maringá, Paraná, Brazil \\ * Author for correspondence: Tel.: +55 443011 4809; Fax: +55 443011 4850; melyssanegri@gmail.com
}

\begin{abstract}
Aim: The present study evaluated the capacity of three species of Fusarium isolated from onychomycosis to form biofilms and the antibiofilm effect of propolis extract on these biofilms. Materials \& methods: The biofilms and antibiofilm effects were evaluated by quantifying the colony-forming units, mitochondrial metabolic activity assays, total biomass by crystal violet staining and scanning electron microscopy. Results: Propolis extract demonstrated significant antibiofilm efficiency on Fusarium spp. isolates and reduced $F$. solani, F. oxysporum and F. subglutinans mature biofilms. Conclusion: Propolis extract can be an alternative topical treatment of onychomycosis caused by Fusarium spp.
\end{abstract}

First draft submitted: 20 March 2017; Accepted for publication: 5 July 2017; Published online: 4 October 2017

Keywords: antifungal $\bullet$ fusariosis $\bullet$ paronychia $\bullet$ treatment $\bullet$ ungueal mycosis

Fusarium species are geophilic fungi, frequently isolated from opportunistic infections in immunocompromised patients, causing disseminated infections with high rates of mortality [1]. Onychomycosis, a fungal infection caused by Fusarium spp., can also affect immunocompetent patients [2]. It has previously been reported that paronychia, a characteristic of onychomycosis caused by this fungus, can be an entry portal to disseminated infections [3]. Moreover, fusariosis has the highest incidence of infection among invasive fungal diseases in Brazil, with high mortality in hematological patients [4].

While the pathogenesis of fusariosis is not completely understood, the virulence factors of these fungi appear to be determinants for infection. Of these, biofilms are related to antifungal resistance and greater difficulty in treatment [5]. Additionally, some studies have described the capacity of $F$. oxysporum to form biofilm in vitro [6] or as an onychomycosis agent [7].

Studies focusing on finding new antifungal agents are urgently required, as there is a limited therapeutic arsenal available for Fusarium spp. infections. Alternatives include natural products, especially as they are inexpensive and have low toxicity. Propolis is a natural product collected from the hives of Apis mellifera L. Several studies have described the medicinal properties of propolis, such as its anti-inflammatory and antitumor effects and antifungal and antibacterial activity [8,9]. In the present study, biofilms formed by three species of Fusarium were characterized and the activity of propolis extract $(\mathrm{PE})$ against these biofilms was evaluated.

\section{Materials \& methods}

Fusarium strains

Fungi were isolated from the nail samples of patients with onychomycosis. Nail scrapings were cultivated in three tubes containing Sabouraud dextrose agar (SDA; Himedia Laboratories, Mumbai, India) and three tubes that contained Mycosel medium (Himedia Laboratories, Mumbai, India). The identification of fungi was performed using classic methods, including the examination of colonies and microscopic morphology [10,11]. The fungi isolated and identified were maintained in a freeze-dried state in the Mycological Collection of the Laboratory of Medical Mycology of the State University of Maringá (UEM), Brazil. One clinical isolate was randomly chosen from each of 
the three most frequent species of Fusarium in our region: F. oxysporum (FO42), F. solani (FS04) and F. subglutinans (FSub39). We also used a reference strain of F solani (ATCC 36031), kindly provided by Fiocruz (Oswaldo Cruz Foundation, Brazil).

\section{Biofilm formation}

This assay was based on the methodology described by Silva et al. [12] with modifications. The strains of Fusarium spp. were grown on potato dextrose agar (PDA; Himedia Laboratories, Mumbai, India) for 7 days at $25^{\circ} \mathrm{C}$. The colonies were gently scraped, harvested in $0.85 \%$ sterile saline and the conidia were counted in a Neubauer chamber. The inoculum was adjusted to a final concentration of $1 \times 10^{6}$ conidia $\mathrm{ml}^{-1}$ [13,14] on Sabouraud dextrose broth medium (SDB; Himedia Laboratories), and $200 \mu \mathrm{l}$ of this suspension was placed into 96-well plates. The plates were then incubated without agitation at $37^{\circ} \mathrm{C}$ for $2 \mathrm{~h}$ in order to settle and to adhere the conidia to the bottom of the plate, and were then incubated at $37^{\circ} \mathrm{C}$ in a shaker at $110 \mathrm{rev} \mathrm{min}^{-1}$. Every $24 \mathrm{~h}, 100 \mu \mathrm{l}$ of SDB was removed and an equal volume of fresh SDB without Fusarium was added to each well for renewal of the culture medium. The plates were then incubated for $72 \mathrm{~h}$. All experiments were repeated on three occasions with individual samples evaluated in triplicate.

\section{Biofilm biomass quantification by crystal violet staining}

Biofilms were allowed to grow for 24, 48 and $72 \mathrm{~h}$. They were then washed three-times with sterile saline and after drying, $200 \mu \mathrm{l}$ of methanol was added to each well for $15 \mathrm{~min}$ to affix the biofilms. Later, $200 \mu \mathrm{l}$ of crystal violet $(1 \% \mathrm{v} / \mathrm{v})$ was added for $5 \mathrm{~min}$. The wells were washed with sterile distilled water and $200 \mu \mathrm{l}$ of acetic acid (33\% $\mathrm{v} / \mathrm{v}$ ) was then added to dissolve the stain. The obtained solution was read in a microtiter plate reader at $570 \mathrm{~nm}$ and the absorbance values were standardized per unit area of well (absorbance $\mathrm{cm}^{-2}$ ).

\section{Biofilm metabolic activity assay by XTT reduction}

The reduction assay of the tetrazolium salt 2,3-(2-methoxy-4-nitro-5-sulphophenyl)-5-([phenylamino]carbonyl)$2 \mathrm{H}$ tetrazolium hydroxide (XTT; Sigma-Aldrich, MO, USA) was used to determine in situ biofilm mitochondrial activity. After biofilm formation, each well was washed three-times with sterile saline. A total of $100 \mu$ l of a solution containing XTT, phenazine methosulphate (PMS) and 0.85\% sterile saline was added to each well: $60 \mu \mathrm{l}$ of sterile saline, then $20 \mu \mathrm{l}$ of XTT stock solution $\left(500 \mu \mathrm{g} \mathrm{ml}^{-1}\right)$ and $20 \mu \mathrm{l}$ of PMS stock solution $\left(50 \mu \mathrm{g} \mathrm{ml}^{-1}\right)$ were added. The final concentrations of XTT and PMS in the wells were 100 and $10 \mu \mathrm{g} \mathrm{ml}^{-1}$, respectively. The plates were then incubated at $35^{\circ} \mathrm{C}$ for $3 \mathrm{~h}$, protected from the light. After this period, the absorbance of the obtained solution was read in a microtiter plate reader at $492 \mathrm{~nm}$ and the absorbance values were standardized per unit area of well (absorbance $\mathrm{cm}^{-2}$ ).

\section{Scanning electron microscopy}

This assay was based on the methodology described by Silva et al. [12]. To examine the structure of biofilms by scanning electron microscopy (SEM), biofilms were allowed to form in 24-well polystyrene microtiter plates as described above for $72 \mathrm{~h}$. The wells were washed with sterile saline and the biofilms were dehydrated with alcohol (using 70\% for $10 \mathrm{~min}, 80 \%$ for $10 \mathrm{~min}, 95 \%$ for $10 \mathrm{~min}$ and $100 \%$ ethanol for $20 \mathrm{~min}$ ) and air dried. Prior to observation, the bases of the wells were cut out and mounted onto aluminum stubs, sputter coated with gold and observed with an S-360 scanning electron microscope (Leo, MA, USA).

\section{Biofilm viability assay}

Biofilms were formed for 24, 48 and $72 \mathrm{~h}$ and were washed three-times with sterile saline. Then, $200 \mu \mathrm{l}$ of saline was added to each well and the biofilms were vigorously scraped with a pipette, transferred to a conical tube and vortexed for $1 \mathrm{~min}$. Aliquots of $15 \mu \mathrm{l}$ were placed in a plate containing Dichloran rose bengal chloramphenicol agar (Merck, Darmstadt, Germany) and were incubated at $35^{\circ} \mathrm{C}$. Dichloran rose bengal chloramphenicol agar is a selective medium for the isolation and enumeration of viable molds and restricts colony size, improving the enumeration and detection of the molds. The number of colony-forming units was determined after 5 days of incubation. 


\section{PE \& phenol content}

The propolis source was as described by Capoci et al. [15], with green propolis collected from hives of $A$. mellifera in Cianorte (Paraná, Brazil). The samples were triturated and stored at $-20^{\circ} \mathrm{C}$ until further analysis. PE was prepared with a propolis/ethanol ratio of $30 / 70(\mathrm{w} / \mathrm{w})$ by turbo extraction, at $3500 \mathrm{rpm}$, three-times at 15 min with two intervals of $5 \mathrm{~min}$. PE was filtered through filter paper and adjusted to the initial weight with ethanol. The total phenol content (TPC) of PE was evaluated by the Folin-Ciocalteau method [16]. PE was added to $6 \mathrm{ml}$ of the Folin-Ciocalteau and $6 \mathrm{ml}$ of $20 \% \mathrm{Na}_{2} \mathrm{CO}_{3}$ (Sigma-Aldrich). After $2 \mathrm{~h}$, absorbance was determined by Shimadzu UV-1650PC spectrophotometer (Tokyo, Japan) at a wavelength of $760 \mathrm{~nm}$. A calibration curve with solutions of gallic acid was used as a reference. TPC was expressed as a percentage of total phenolic substances in PE and corresponded to a mean of six replicates.

\section{Antifungal assay}

In vitro antifungal susceptibility testing of planktonic cells was performed using a microdilution method adapted from Capoci et al. [15] and the Clinical and Laboratory Standards Institute M38-A2 protocol [17] with serial dilution of PE 34.17, 68.35, 136.71, 273.43, 546.87, 1093.75, 2187.5, 4375, 8750 and 17,500 $\mathrm{gg} \mathrm{ml}^{-1}$ of TPC expressed in gallic acid in RPMI 1640 (Roswell Park Memorial Institute; Gibco, NY, USA) with l-glutamine (with sodium bicarbonate) and 0.165 M 3-(N-morpholino) propanesulfonic acid (pH 7.2) as a buffer (Sigma-Aldrich). The strains of Fusarium spp. were grown on potato dextrose agar for 7 days at $25^{\circ} \mathrm{C}$. The colonies were gently scraped and harvested in $0.85 \%$ sterile saline solution. The number of conidia were counted in a Neubauer chamber and the inoculum was adjusted to a final concentration of $5 \times 10^{4}$ conidia $\mathrm{ml}^{-1}$ in RPMI 1640. An aliquot of PE $(100 \mu \mathrm{l})$ was dispensed into 96-well plates and further incubated with aliquots $(100 \mu \mathrm{l})$ of the Fusarium inoculum. The 96-well plates were incubated at $35^{\circ} \mathrm{C}$ for $72 \mathrm{~h}$. The minimal inhibitory concentration (MIC) values were determined by visualization, corresponding to the lowest concentration of antifungal agent in which no visible growth was observed, in comparison with the control (cells grown without PE) [15,17]. The minimal fungicidal concentration (MFC) was determined after $72 \mathrm{~h}$ of incubation at $35^{\circ} \mathrm{C}$ and $5 \mu \mathrm{l}$ of each well was transferred to a plate containing sabouraud dextrose agar and incubated at $25^{\circ} \mathrm{C}$ for $48 \mathrm{~h}$.

\section{Antibiofilm assay}

Once stable and mature biofilms were formed (for $24 \mathrm{~h}$ as described above) they were washed three-times with sterile saline. Biofilms were formed as described above for $24 \mathrm{~h}$. Then, PE was added at the MFC concentration found in the latter assay. The 96 -well plates were incubated at $37^{\circ} \mathrm{C}$ for a further $24 \mathrm{~h}$. The activity of PE against biofilms was determined by biomass quantification using crystal violet staining and by biofilm viability assay described above.

\section{Epifluorescence microscopy}

Antibiofilm assay was also evaluated by epifluorescence microscopy. Biofilms were formed in 24-well polystyrene microtiter plates as described above for $24 \mathrm{~h}$, then PE was added at the MFC concentration and the plates were incubated at $37^{\circ} \mathrm{C}$ for a further $24 \mathrm{~h}$. The matrix of biofilms was then stained with calcofluor white (Fluka Analytical, MO, USA) diluted in sterile saline (1:4) for $5 \mathrm{~min}$ and washed twice with sterile saline. Stained biofilms were observed with a microscope (Evos FL, Live Technologies, CA, USA) with filters capable of detecting the fungi cell wall (BP 365-370, FT 400, LP 421).

\section{Statistical analysis}

Data were analyzed using Prism 6.0 software (GraphPad, CA, USA). The $t$-test and one-way analysis of variance with Bonferroni were used. All of the tests were performed with a confidence level of $95 \%$ and values of $\mathrm{p} \leq 0.05$ were considered statistically significant.

\section{Results}

The three species of Fusarium tested in this study formed biofilms. Nevertheless, the F. solani strains were more efficient than the other species in relation to total biomass and the number of viable cells. Furthermore, the biomass produced by both the clinical isolates and reference strain of this species was directly proportional to incubation time (Figure 1). While the total biomass of biofilms formed by F. solani increased from 24 to $48 \mathrm{~h}$ and from 48 to $72 \mathrm{~h}$ of incubation, the total biomass of F. oxysporum and F. subglutinans biofilms increased only until $48 \mathrm{~h}$ 


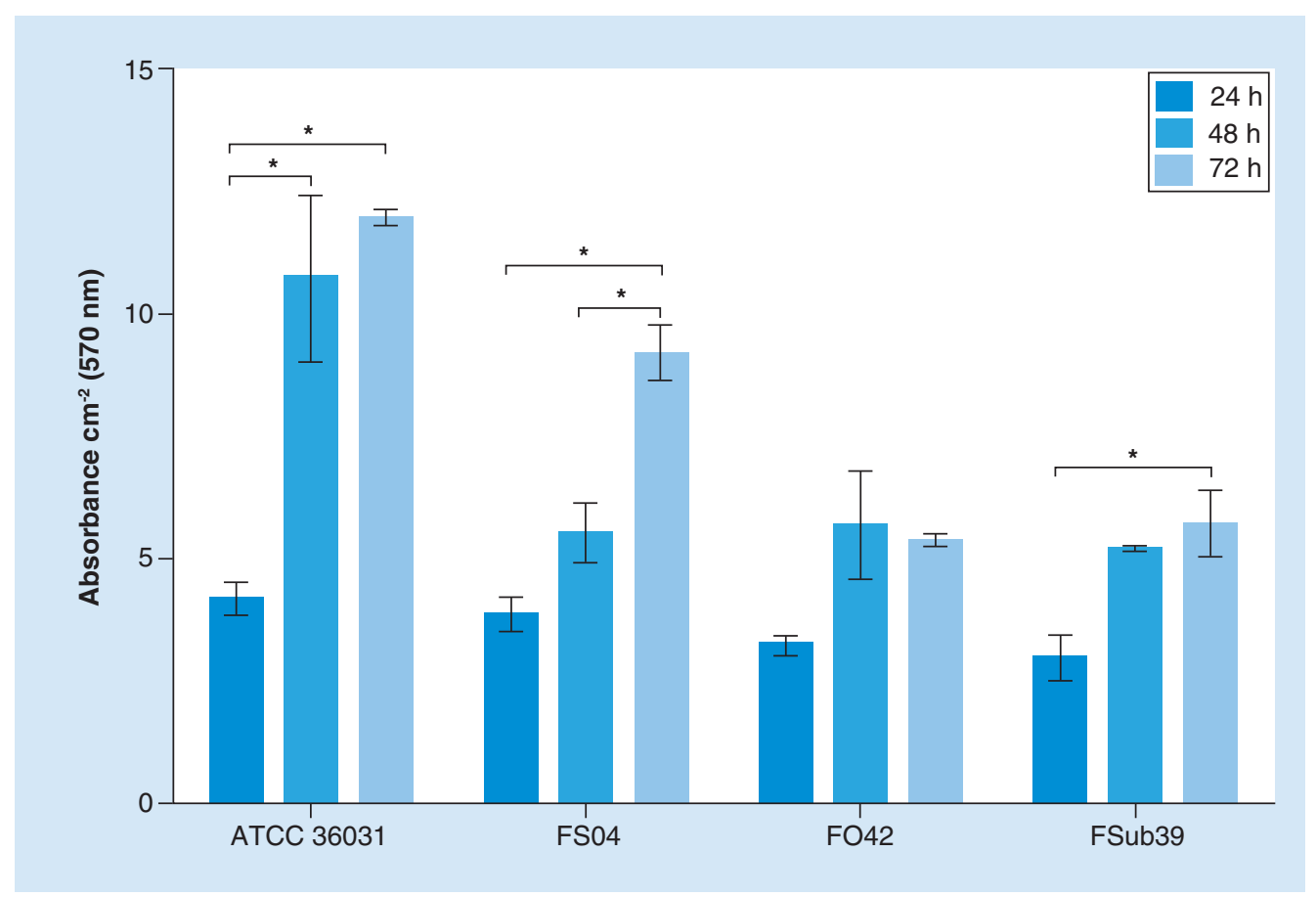

Figure 1. Crystal violet staining of biofilm biomass of Fusarium solani (ATCC 36031 and FS04), Fusarium oxysporum (FO42) and Fusarium subglutinans (FSub39) at different times of incubation. ANOVA with Bonferroni, * $p<0.05$.

and apparently became stable after this period (Figure 1). However, these increases in biomass production were significant only for strains of $F$. solani and F. subglutinans, and mainly from 24 to $72 \mathrm{~h}$ (Figure 1).

In contrast, the absorbance values from the XTT reduction assay, related to biofilm metabolic activity, decreased with incubation time. The decrease in absorbance values was significant from 24 to $72 \mathrm{~h}$ for all the isolates (Figure 2).

The clinical isolate of $F$ solani also presented significantly more viable cells on the biofilm than the other species at 48 and $72 \mathrm{~h}$ of incubation. Most isolates, except for F. oxysporum, exhibited a significant increase in viable cells from 24 to $48 \mathrm{~h}$ and from 24 to $72 \mathrm{~h}$ (Figure 3). The number of viable cells and the quantity of biomass were quite similar among species with $24 \mathrm{~h}$ of incubation, but after this period, they began to show differences among themselves.

SEM analysis revealed that the morphologies of the biofilm structure formed by the three different Fusarium species differed. It was observed that biofilms of $F$. solani were formed by narrow, smooth hyphae; and conidiophores with monophiliades, as well as macroconidia, were found in the clinical isolate (FS04). The biofilm of $F$. oxysporum was formed by smooth hyphae with larger lumen, while $F$. subglutinans exhibited crystal-encrusted hyphae. The structure of biofilms examined by SEM is shown in Figure 4.

Regarding in vitro antifungal susceptibility testing, PE exhibited strong fungicidal activity against Fusarium spp. isolates in planktonic cells. The MIC50 (i.e., the MIC that inhibited 50\% of the isolates tested) and MIC90 (i.e., the MIC that inhibited $90 \%$ of the isolates tested) corresponded to $1093.75 \mathrm{\mu g} \mathrm{m}^{-1}$ of TPC expressed in gallic acid and was used to test the fungicide activity of PE against biofilms formed by Fusarium species. Furthermore, MIC and MFC were coincident (1093.75 $\mathrm{\mu g} \mathrm{ml}^{-1}$ of TPC expressed in gallic acid) for all species.

In the exposure of the biofilms to PE, it was observed that the biomass of those treated with PE was significantly lower than control (Figure 5). Similarly, the number of viable cells also decreased significantly after exposure to PE (Figure 6). These results were confirmed by epifluorescence microscopy, in which it was observed that the number of cells of biofilms treated with PE decreased and were more disorganized and damaged than control, without PE (Figure 7). These data demonstrated the antifungal activity of PE not only on planktonic cells but also on Fusarium biofilms. 


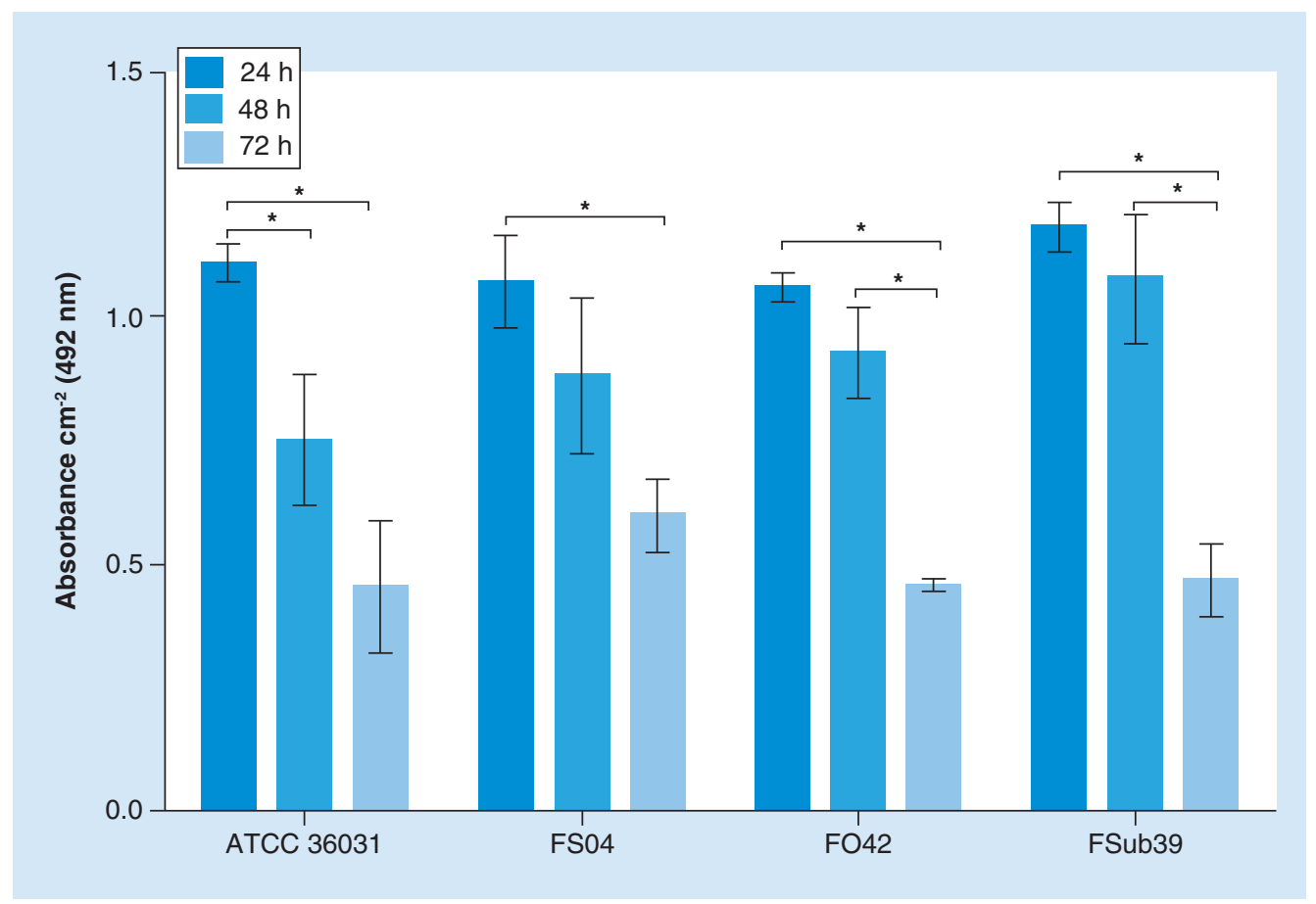

Figure 2. Absorbance values of XTT reduction assay of the three clinical isolates from onychomycosis and a reference strain (Fusarium solani ATCC 36031) biofilms at different times of incubation. ANOVA with Bonferroni, ${ }^{*} \mathrm{p}<0.05$.

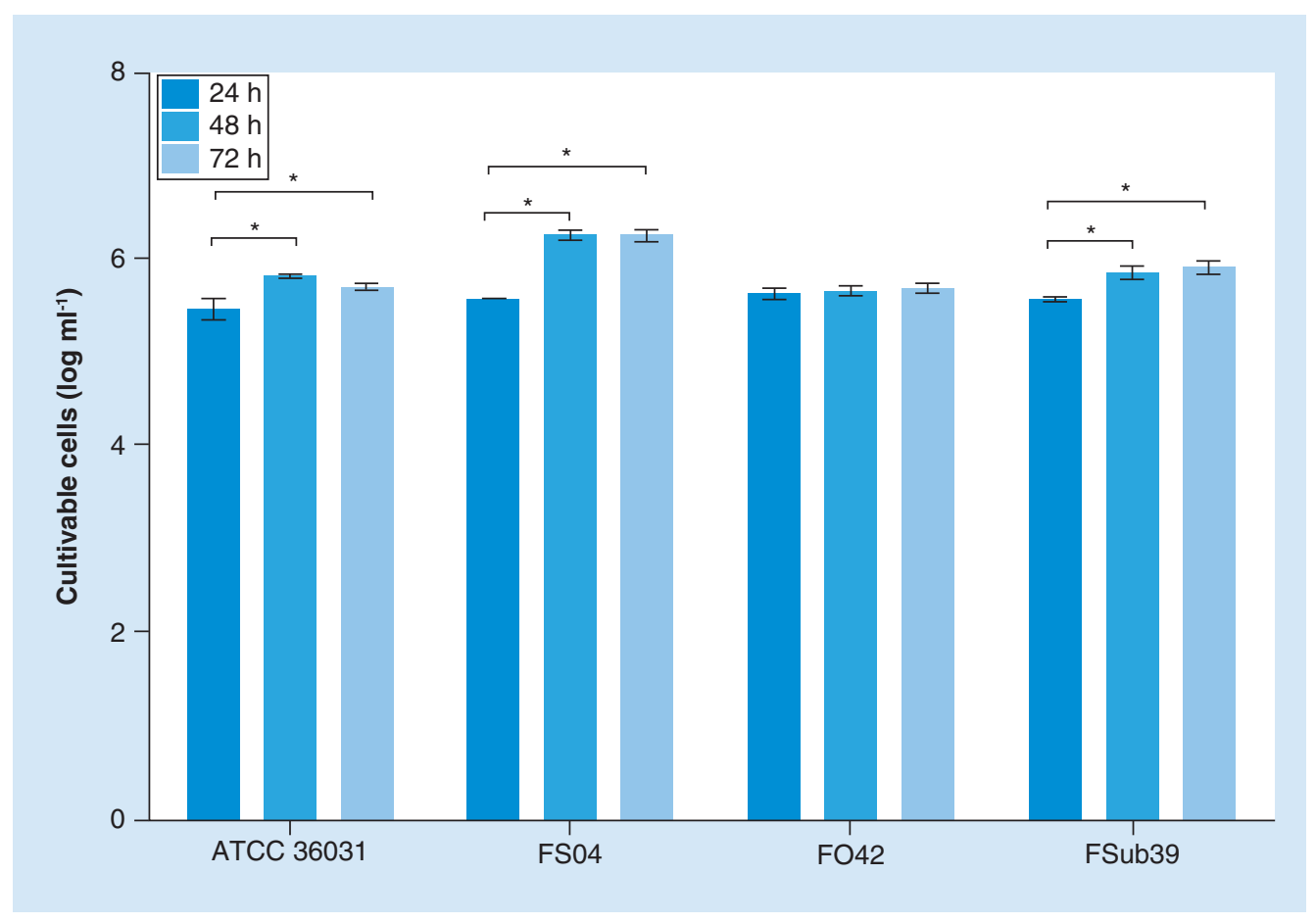

Figure 3. Colony forming units from Fusarium solani (ATCC 36031 and FS04), Fusarium oxysporum (FO42) and Fusarium subglutinans (FSub39) biofilms at different times of incubation. ANOVA with Bonferroni, * $p<0.05$. 


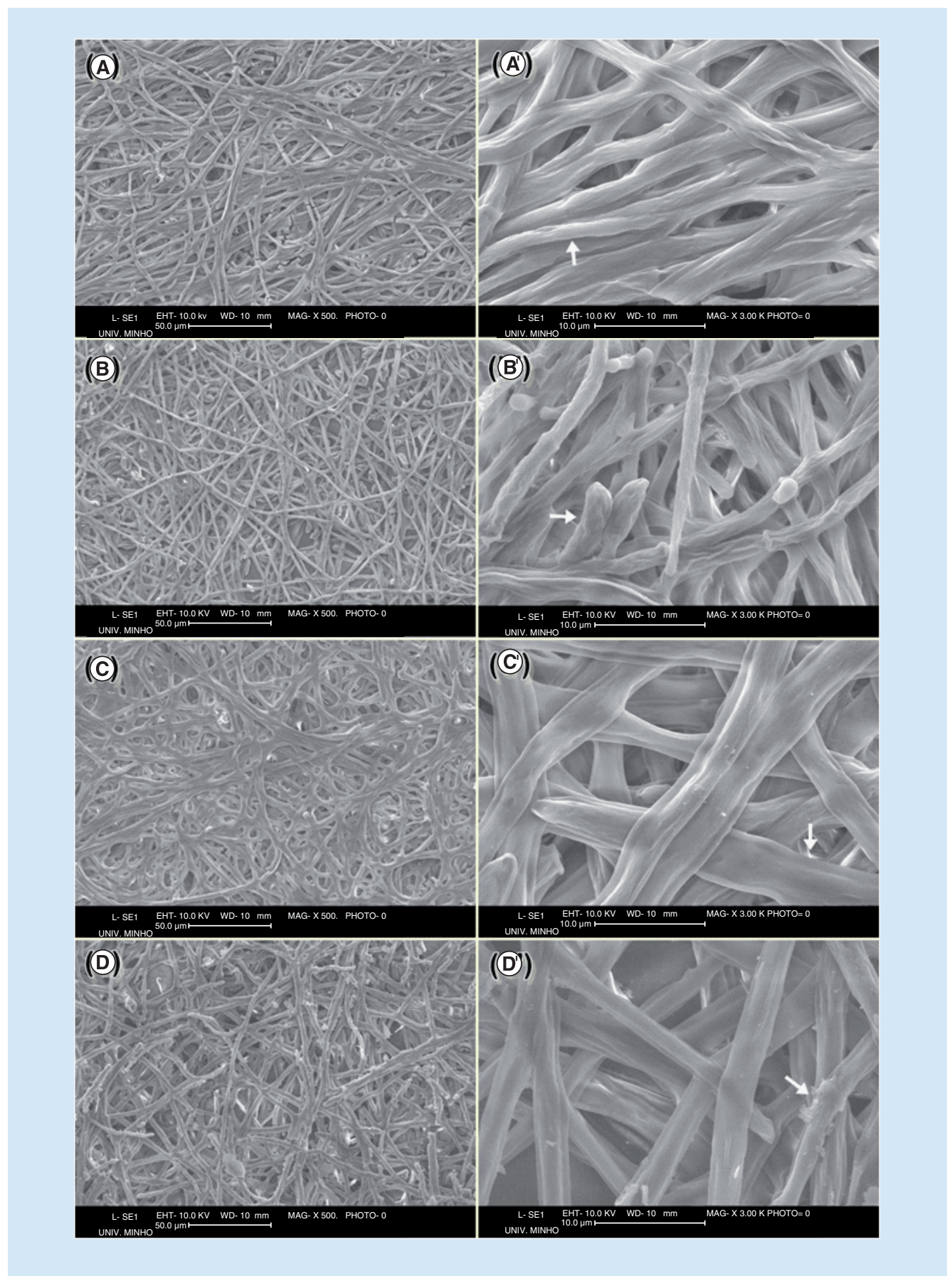

Figure 4. Scanning electron microscopy of Fusarium solani ATCC 36031 (A, A'), Fusarium solani FS04 (B, B'), Fusarium oxysporum FO42 (C, $\left.C^{\prime}\right)$ and Fusarium subglutinans FSub39 $\left(D, D^{\prime}\right)$ biofilms with $72 \mathrm{~h}$ of incubation. First column $500 \times$ magnification $(A, B, C, D)$, second column $3000 \times$ magnification $\left(A^{\prime}, B^{\prime}, C^{\prime}, D^{\prime}\right)$.

\section{Discussion}

Onychomycosis caused by Fusarium spp. is frequent in Brazil and has been increasing in recent years [2,10]. Its pathogenesis and treatment, however, have not yet been completely elucidated.

According to our previous study [2], onychomycosis caused by Fusarium spp. is characterized by painful lesions, paronychia and inflammatory reaction. In addition, the primary skin lesions caused by Fusarium spp. have been considered a portal of entry for disseminated infections caused by this fungus, especially in neutropenic and 


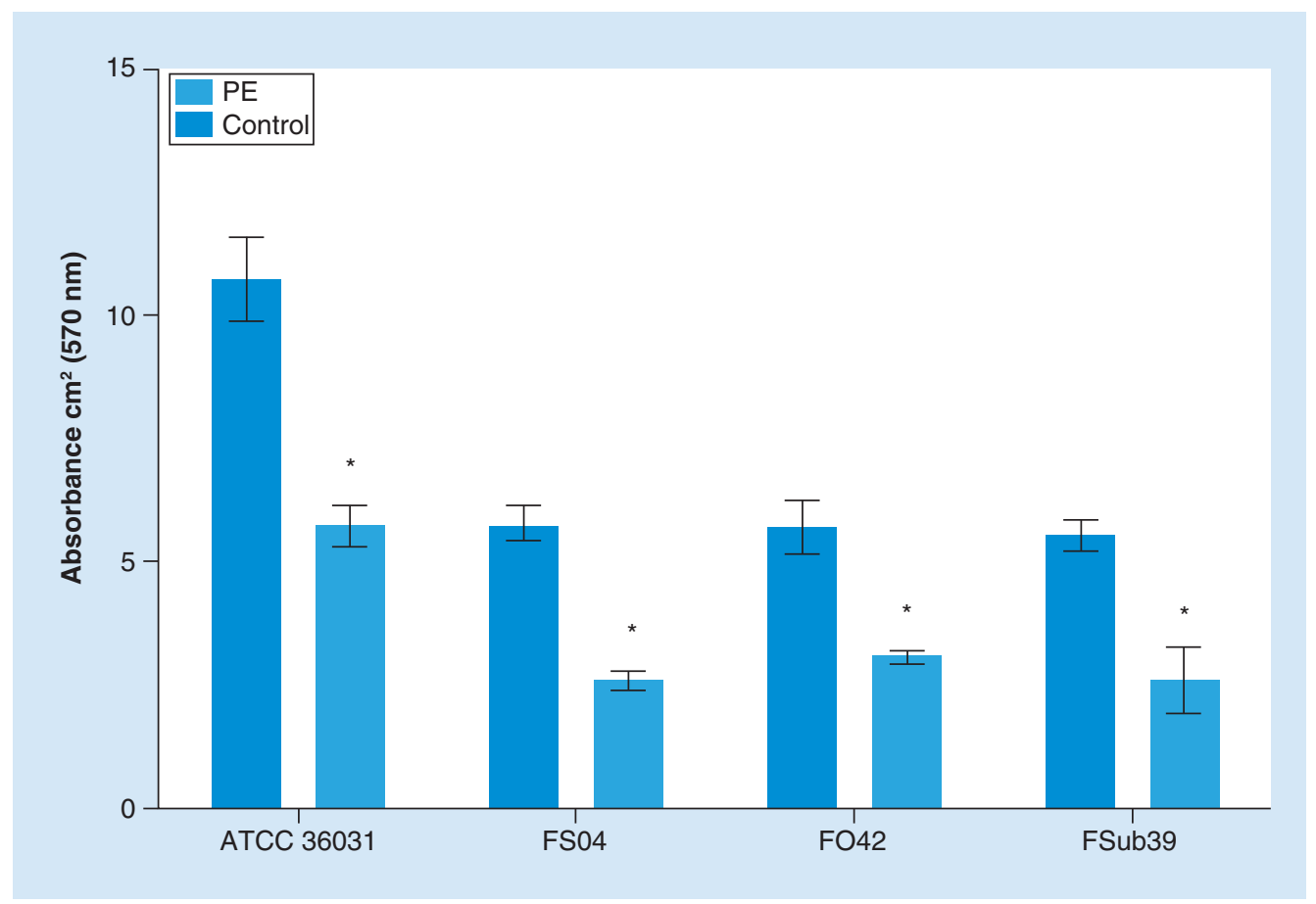

Figure 5. Crystal violet staining of biofilm biomass of Fusarium solani (ATCC 36031 and FS04), Fusarium oxysporum (FO42) and Fusarium subglutinans (FSub39) exposed to propolis extract and control, without propolis extract. $t$-test, $* p<0.05$.

PE: Propolis extract.

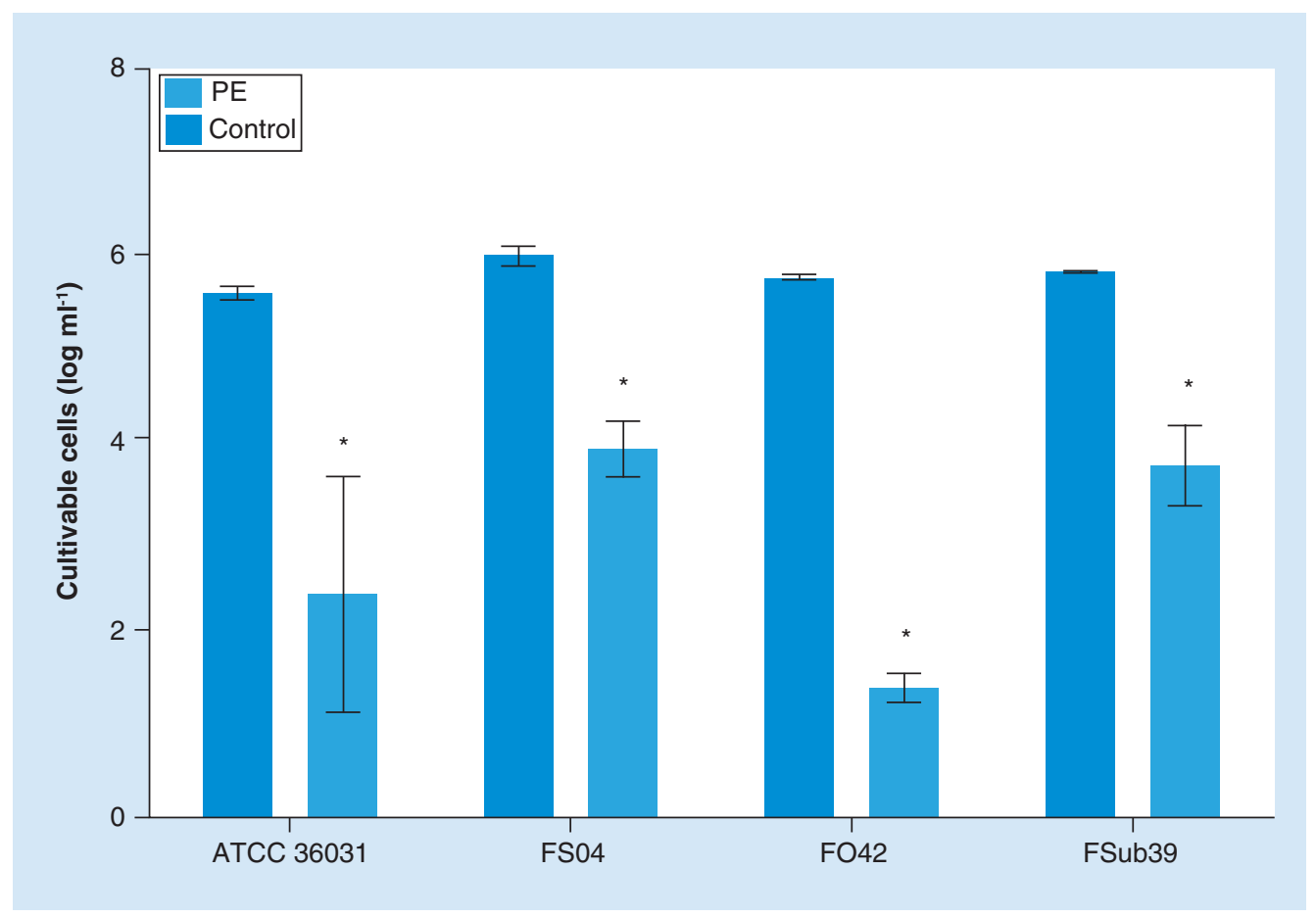

Figure 6. Colony forming units from Fusarium solani (ATCC 36031 and FS04), Fusarium oxysporum (FO42) and Fusarium subglutinans (FSub39) biofilms exposed to propolis extract and control.

$t$-test, ${ }^{*} p<0.05$

PE: Propolis extract. 


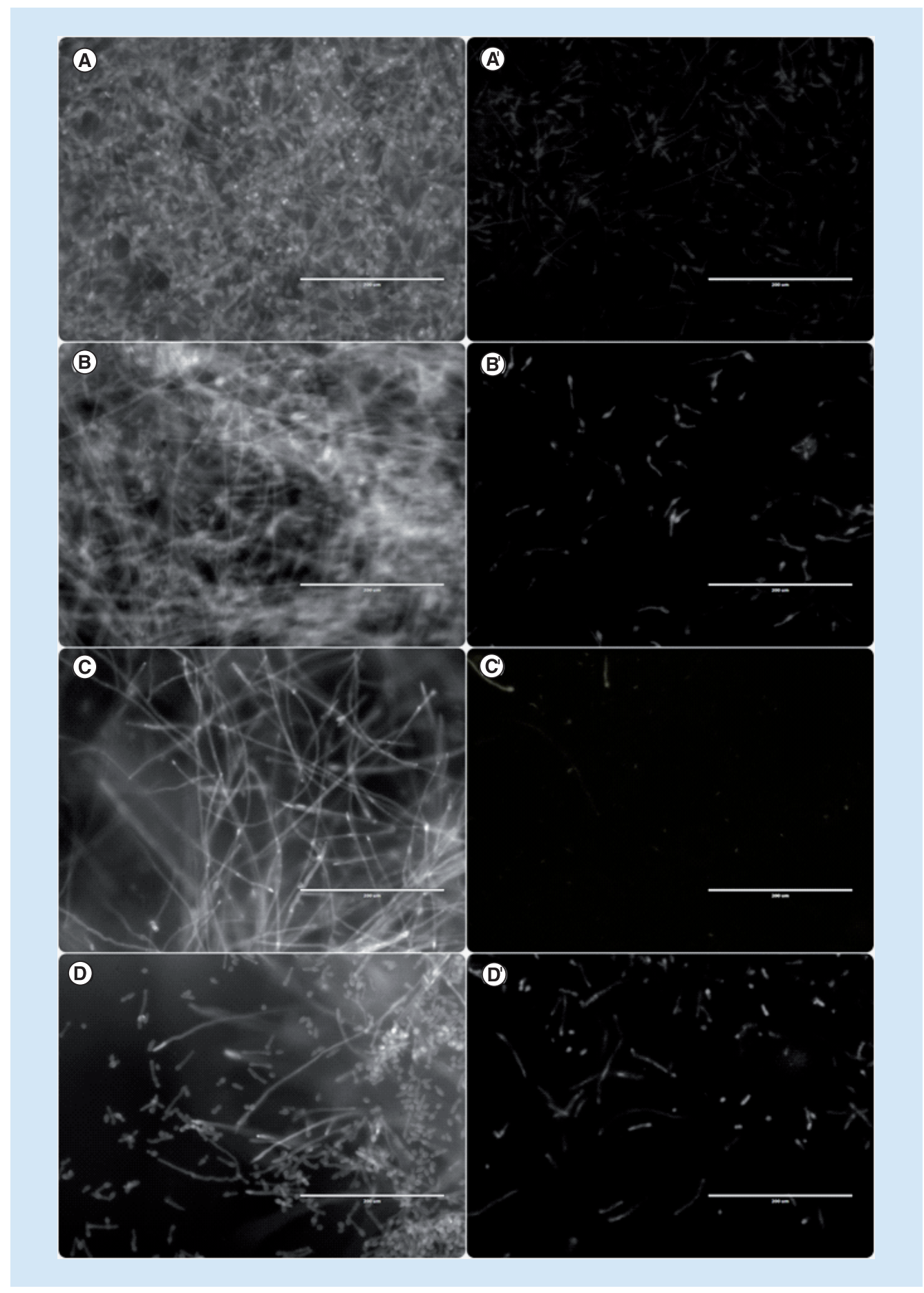

Figure 7. Epifluorescence of Fusarium solani ATCC 36031 (A, A'), Fusarium solani FS04 (B, B'), Fusarium oxysporum FO42 (C, $\left.C^{\prime}\right)$ and Fusarium subglutinans FSub39 (D, $\left.D^{\prime}\right)$. First column shows control without $P E, 2000 \times$ magnification $(A, B, C, D)$, second column shows biofilms treated with $P E, 2000 \times$ magnification $\left(A^{\prime}, B^{\prime}, C^{\prime}, D^{\prime}\right)$. PE: Propolis extract.

transplanted patients [3,18]. Although Fusarium spp. is considered a non-keratinolytic fungus, this genus is able to cause onychomycosis with the ability to form biofilms. According to Gupta et al. [7], there is evidence that in onychomycosis, the fungi may be arranged in biofilms in complex, sessile microbial communities attached 
to epithelial surfaces. The capacity of $F$. oxysporum to form biofilms on fragments of human nails has also been documented [6].

It has recently been shown for the first time that Fusarium spp. can grow in vitro using human nails as a single source of nutrient; and also that members from this genus have the ability to invade healthy human nails [10]. In the current study, the capacity of clinical isolates of F. solani, F. oxysporum and F. subglutinans obtained from onychomycosis to form biofilms was demonstrated, a finding similar to those described by Costa-Orlandi et al. (2014) [14] for the dermatophytes T. rubrum and T. mentagrophytes, which are the most common agents of onychomycosis.

While all the species of Fusarium tested in this study formed biofilms, the F. solani strains were more efficient than the others for all the studied parameters. The results of total biomass quantification and viable cell characterization revealed the greater capacity of F solani to form biofilms (Figures $1 \& 2$ ), suggesting that this species is more virulent than the others. This finding is in accordance with the fact that this species is most frequently isolated from invasive fusariosis [19]. Other studies have also described $F$. solani as the most virulent species, such as when $F$. solani was injected intravenously and caused the death of all the animals tested, which other Fusarium species did not $[20]$.

Curiously, the values of XTT reduction assay in the present study decreased over time (Figure 2), suggesting a reduction in the metabolic activity of the biofilms. It is important to point out that according to biofilm structure, the fungal cells in sessile populations may display a poor physiological state, as confirmed by XTT-based assay [21]. Furthermore, Fusarium biofilms exhibit opposing kinetics (Figures $1 \& 2$ ), as metabolic activity was lowest at the moment of peak biomass production and the inverse effect was also observed, corroborating the findings of other studies [22,23]. This low metabolic activity could be due to the increased adsorption of compounds on the biofilm matrix, meaning there is less contact between the tetrazolium salt and the cells of the mature biofilm [21-23]. It is also important to emphasize that the association between these parameters (low activity and high biomass) is well established in literature and is considered responsible for biofilm antifungal resistance, as fungi arranged in biofilms are considered to be up to 1000 -fold more resistant to antifungal agents than planktonic cells [21].

Regarding fungal biofilm formation, although most attention has been paid to the yeast pathogen Candida albicans, it is known that many fungal species, including filamentous fungi, can form such structures [24,25]. Biofilm morphology can vary according to the species involved and it has been found that even species from the same genus can form biofilms with distinct arrangements [14]. The SEM results of the present study (Figure 4) have shown that the Fusarium species used here can form biofilms with different morphologies and all the isolates were able to organize dense growth, with a well-structured community constituted by large hyphae, sometimes with conidiophores and conidia, suggesting a mature ecosystem.

When considering its ability to form biofilms, Peiqian et al. [13] reported that F. oxysporum when found in biofilms was less susceptible to cold, heat, UV light and some fungicides than those grown in planktonic (free-living) cells. It is well known that the capacity to form biofilms is related to higher resistance and difficulty in clinical treatment [5]. Considering that onychomycosis can be a portal of entry for invasive fusariosis, especially in immunocompromised patients, the search for effective new topical treatments for onychomycosis caused by Fusarium spp. is highly relevant. In addition, few antifungal agents are efficacious against fungal biofilms (echinocandins and liposomal amphotericin B) and these drugs are not used for onychomycoses [21].

In this way, it is important to evaluate if a new antifungal option for planktonic cells exists, as PE also acts against fungi organized in biofilms. Propolis is a resin that presents a number of medicinal properties [8], including anti-inflammatory activity, which could potentiate a possible antifungal topical action. Furthermore, the capacity of propolis to inhibit the biofilm formation of $C$. albicans has already been reported, along with the low cytotoxicity in human cells with the same PE used in the present study [15,26]. However, the antifungal property of PE has not yet been proved in biofilms formed by Fusarium species.

While microbiologists have historically tested the effect of drugs on planktonic cells, the present study evaluated the action of PE on both planktonic cells and biofilm formation by Fusarium species. Initially, good results were obtained for the MIC and MFC of PE against Fusariun spp. planktonic cells, which were $1093.75 \mu \mathrm{g} \mathrm{m}{ }^{-1}$, similar to those described by Dota et al. [27] for Candida spp. The majority of isolates tested had MIC values between 550.3 and $1100.63 \mathrm{\mu g} \mathrm{ml}^{-1}$. Capoci et al. [15] also found similar values of MFC and MIC for Candida, proving that propolis possesses a fungicide mechanism.

Next, the performance of this PE on biofilms formed by the Fusarium species was evaluated. The results were exciting, as PE appears to be efficient, significantly reducing both the biomass and the number of viable 
cell parameters $(\mathrm{p}<0.05)$ of the biofilms produced by all the evaluated isolates (Figures $5 \& 6$ ). In addition, epifluorescence assay showed clearly that the number of cells of the biofilms formed by all the strains treated with PE were reduced and the structure of the biofilm was lost, leaving only some damaged and isolated cells (Figure 7).

A possible limitation of this study was the absence of a comparison between the antibiofilm effects of propolis and conventional antifungals. However, it should be pointed out that other works carried out by our group [15,26] have shown propolis to be as or more efficient than other antifungal drugs. Moreover, Fusarium spp. are highly resistant to azole antifungals which are not usually active against this fungus and terbinafine response varies according to species [10]. We have not therefore included a conventional antifungal as a control.

In summary, it is known that biofilm-forming capacity is related to greater resistance and difficulty in treatment [21]. PE is therefore promising as propolis can be found in its extract form in pharmacies, ready for topical use in patients with onychomycosis, justifying the importance of the development of new treatment approaches with the capacity to control fungal biofilms present in nails affected by Fusarium spp.

\section{Conclusion}

The results of the present study described the biofilm-forming capacity of three species of Fusarium isolated from onychomycosis, with $F$ solani exhibiting the greatest such capacity, reinforcing that it is more virulent than the other Fusarium species tested. We also demonstrated the antifungal and antibiofilm capacity of PE against clinical isolates of F. solani, F. oxysporum and F. subglutinans. This reinforces the previous results and suggests that PE could be an alternative option for the topical treatment of onychomycosis and avoid the possible dissemination of this fungi of onychomycosis, since the most frequent species that cause human invasive fusariosis, $F$. solani and $F$. oxysporum, are also the most frequent species that cause onychomycosis.

\section{Acknowledgements}

The authors thank Fiocruz for kindly providing the strain of Fusarium solani.

\section{Financial \& competing interests disclosure}

This study was supported by grants from Conselho Nacional de Desenvolvimento Científico e Tecnológico (CNPq) and Coordenação de Aperfeiçoamento de Pessoal de Nível Superior (CAPES). The authors have no other relevant affiliations or financial involvement with any organization or entity with a financial interest in or financial conflict with the subject matter or materials discussed in the manuscript apart from those disclosed.

No writing assistance was utilized in the production of this manuscript.

\section{Ethical conduct of research}

The authors state that they have obtained appropriate institutional review board approval or have followed the principles outlined in the Declaration of Helsinki for all human or animal experimental investigations. In addition, for investigations involving human subjects, informed consent has been obtained from the participants involved.

\section{Summary points}

- Fusarium species isolated from onychomycosis form biofilm efficiently.

- Propolis presents potential antifungal activity.

- Propolis is a strong inhibitor of Fusarium spp. from onychomycosis.

- Propolis is able to reduce and destroy biofilms formed by Fusarium species.

- Propolis is a promising alternative to traditional antifungal therapy.

\section{References}

Papers of special note have been highlighted as: $\bullet$ of interest; $\bullet \bullet$ of considerable interest

1. Nucci M, Anaissie E. Fusarium infections in immunocompromised patients. Clin. Microbiol. Rev. 20(4), 695-704 (2007).

2. Guilhermetti E, Takahachi G, Shinobu CS, Svidzinski TIE. Fusarium spp. as agents of onychomycosis in immunocompetent hosts. Int. J. Dermatol. 46(8), 822-826 (2007).

-. Excellent article for understanding Fusarium species infections.

3. Bourgeois GP, Cafardi JA, Sellheyer K, Andea AA. Disseminated Fusarium infection originating from paronychia in a neutropenic patient: a case report and review of the literature. Cutis 85(4), 191-194 (2010). 
4. Nucci M, Garnica M, Gloria $\mathrm{AB}$ et al. Invasive fungal diseases in haematopoietic cell transplant recipients and in patients with acute myeloid leukaemia or myelodysplasia in Brazil. Clin. Microbiol. Infect. 19(8), 745-751 (2013).

5. Mukherjee PK, Chandra J, Yu C, Sun Y, Pearlman E, Ghannoum MA. Characterization of fusarium keratitis outbreak isolates: contribution of biofilms to antimicrobial resistance and pathogenesis. Investig. Opthalmol. Vis. Sci. 53(8), 4450-4457 (2012).

6. Vila TVM, Rozental S, de Sá Guimarães CMD. A new model of in vitro fungal biofilms formed on human nail fragments allows reliable testing of laser and light therapies against onychomycosis. Lasers Med. Sci. 30(3), 1031-1039 (2015).

7. Tamura NK, Kira G, Patussi EV, Donatti L, Inez T, Svidzinski E. Adherence and biofilm formation of Fusarium oxysporum isolated from a corneal ulcer. Glob. Adv. Res. J. Med. Med. Sci. 4(1), 28-34 (2015).

8. Burdock GA. Review of the biological properties and toxicity of bee propolis (propolis). Food Chem. Toxicol. 36(4), 347-363 (1998).

9. Negri M, Salci T, Shinobu-Mesquita C, Capoci I, Svidzinski T, Kioshima E. Early state research on antifungal natural products. Molecules 19(3), 2925-2956 (2014).

10. Galletti J, Negri M, Grassi FL, Kioshima-Cotica ÉS, Svidzinski TIE. Fusarium spp. is able to grow and invade healthy human nails as a single source of nutrients. Eur. J. Clin. Microbiol. Infect. Dis. 34(9), 1767-1772 (2015).

- Excellent standard for understanding the pathogenicity of Fusarium.

11. De Hoog GS, Guarro J. Atlas of Clinical Fungi. Centraalbureau voor Schimmelcultures, Reus, Spain (1995).

12. Silva S, Henriques M, Martins A, Oliveira R, Williams D, Azeredo J. Biofilms of non-Candida albicans Candida species: quantification, structure and matrix composition. Med. Mycol. 47(7), 681-689 (2009).

13. Peiqian L, Xiaoming P, Huifang S, Jingxin Z, Ning H, Birun L. Biofilm formation by Fusarium oxysporum f. sp. cucumerinum and susceptibility to environmental stress. FEMS Microbiol. Lett. 350(2), 138-145 (2014).

14. Costa-Orlandi CB, Sardi JCO, Santos CT, Fusco-Almeida AM, Mendes-Giannini MJS. In vitro characterization of Trichophyton rubrum and T. mentagrophytes biofilms. Biofouling 30(6), 719-727 (2014).

15. Capoci GRI, Bonfim-mendonça PDS, Arita GS et al. Propolis is an efficient fungicide and inhibitor of biofilm production by vaginal Candida albicans. Evid. Based Complement Alternat. Med. 2015, 28769 (2015).

- Excellent article for understanding the potential of propolis extract.

16. Pereira RRA, Bruschi ML. Evaluation of two spectrophotometric methods for analysis of green propolis extract. Lat. Am. J. Pharm. 32(5), 719-726 (2013).

17. Clinical and Laboratory Standards Institute Reference Method for Broth Dilution Antifungal Susceptibility Testing of Filamentous Fungi (2nd Edition). Clinical and Laboratory Standards Institute, PA, USA, 1-35 (2008).

18. Nucci M, Varon AG, Garnica $M$ et al. Increased incidence of invasive fusariosis with cutaneous portal of entry, Brazil. Emerg. Infect. Dis. 19(10), 1567-1572 (2013).

19. Muhammed C, Anagnostou T, Desalermos A et al. Fusarium infection: report of 26 cases and review of 97 cases from the literature. Medicine 92(6), 305-316 (2013).

- Excellent article for understanding Fusarium infections.

20. Mayayo E, Pujol I, Guarro J. Experimental pathogenicity of four opportunist Fusarium species in a murine model. J. Med. Microbiol. 48(4), 363-366 (1999).

21. Ramage G, Rajendran R, Sherry L, Williams C. Fungal biofilm resistance. Int. J. Microbiol. 2012, 528521 (2012).

22. Marcos-Zambrano LJ, Escribano P, Bouza E, Guinea J. Production of biofilm by Candida and non-Candida spp. isolates causing fungemia: comparison of biomass production and metabolic activity and development of cut-off points. Int. J. Med. Microbiol. 304(8), 1192-1198 (2014).

- Excellent standard for understanding the biofilm cut-off points.

23. Negri M, Silva S, Capoci IRG, Azeredo J, Henriques M. Candida tropicalis biofilms: biomass, metabolic activity and secreted aspartyl proteinase production. Mycopathologia 181(3-4), 217-224 (2016).

24. Sardi JDCO, Pitangui NDS, Rodríguez-Arellanes G, Taylor ML, Fusco-Almeida AM, Mendes-Giannini MJS. Highlights in pathogenic fungal biofilms. Rev. Iberoam. Micol. 31(1), 22-29 (2014).

25. Imamura Y, Chandra J, Mukherjee PK et al. Fusarium and Candida albicans biofilms on soft contact lenses: model development, influence of lens type, and susceptibility to lens care solutions. Antimicrob. Agents Chemother. 52(1), 171-182 (2008).

26. Tobaldini-Valerio FK, Bonfim-Mendonça PS, Rosseto HC et al. Propolis: a potential natural product to fight Candida species infections. Future Microbiol. 11, 1035-1046 (2016).

27. Dota KFD, Consolaro MEL, Svidzinski TIE, Bruschi ML. Antifungal activity of Brazilian propolis microparticles against yeasts isolated from vulvovaginal candidiasis. Evid. Based Complement Alternat. Med. 2011, 201953 (2011). 
